\title{
Expresión de IL-10, IL-4 e IFN-y en lesiones activas de piel en niños con urticaria papular por picadura de pulga
}

\author{
Elizabeth García1 ${ }^{1}$ Silvia Duarte², Camila Calderón ${ }^{3}$, John Mario González4, Adriana Cuéllar², \\ Alberto Gómez ${ }^{5}$, Evelyne Halpert ${ }^{6}$, Adriana Rodríguez $^{2,7}$ \\ 1 Sección de Alergia Pediátrica, Fundación Santa Fe de Bogotá, Bogotá, D.C., Colombia \\ 2 Facultad de Medicina, Universidad Militar Nueva Granada, Bogotá, D.C., Colombia \\ ${ }^{3}$ Grupo de Inmunobiología y Biología celular, Facultad de Ciencias, Pontificia Universidad Javeriana, Bogotá, \\ D.C., Colombia \\ ${ }^{4}$ Grupo de Ciencias Básicas Médicas, Facultad de Medicina, Universidad de los Andes, Bogotá, D.C., Colombia \\ ${ }^{5}$ Instituto de Genética Humana, Facultad de Medicina, Pontifica Universidad Javeriana, Bogotá, D.C., Colombia \\ ${ }^{6}$ Sección de Dermatología Pediátrica, Fundación Santa Fe de Bogotá, Bogotá, D.C., Colombia \\ 7 Facultad de Odontología, Pontificia Universidad Javeriana, Bogotá, D.C., Colombia \\ El trabajo se realizó en la Fundación Santa Fe de Bogotá y la Universidad de los Andes.
}

Introducción. La urticaria papular por picadura de pulga se conoce como una enfermedad alérgica. Sin embargo, las investigaciones no muestran una clara relación con las enfermedades alérgicas.

Objetivo. Estudiar la expresión de IL-10, IL-4 e IFN-y, como marcadores de la respuesta efectora de células T en lesiones de piel de pacientes con urticaria papular por picadura de pulga.

Materiales y métodos. Se incluyeron 14 biopsias de lesiones de piel de niños con diagnóstico de urticaria papular por picadura de pulga y 5 biopsias de piel sana obtenidas de niños sometidos a cirugía por enfermedades no inflamatorias. Todas las muestras se obtuvieron de niños menores de 12 años. Se extrajo ARN con trizol y se cuantificaron los niveles de expresión de las citocinas con la técnica de reacción en cadena de la polimerasa en tiempo real.

Resultados. En los pacientes con urticaria papular por picadura de pulga, se encontró amplia diversidad en los niveles de expresión de IFN-y e IL-10, y valores bajos constantes para IL-4. Se observaron tres perfiles que no corresponden a un patrón común en los pacientes. Las muestras obtenidas de tejidos sanos no presentaron expresión de las citocinas.

Conclusiones. Los datos corresponden a la primera descripción de citocinas que median la respuesta inmunitaria en el sitio de la lesión cutánea en niños con con urticaria papular por picadura de pulga, lo cual indica que la respuesta local es mixta ya que no se encuentra predominio de un fenotipo específico en ninguno de los pacientes.

Palabras clave: urticaria, citocinas, interleucina-4, interleucina-10, interferón gamma.

\section{Expression of IL-10, IL-4 and IFN-y in active skin lesions of children with papular urticaria}

Introduction: Papular urticaria caused by the bites of fleas traditionally has been defined as a chronic allergic disease. However, currently no clear relationship has been described between this pathology and common allergic diseases.

Objective: The expression of IL-10, IL-4 and IFN-y as markers of effector T cell responses was examined in skin lesions of patients with papular urticaria by flea bite.

Materials and methods: Fourteen skin lesion biopsies were sampled from children with a clinical diagnosis of papular urticaria by flea bite and were compared with 5 healthy skin biopsies of children

\footnotetext{
Colaboración de los autores:

Elizabeth García: diseño del estudio, consecución de recursos de la Fundación Santa Fe de Bogotá, administración de recursos financieros, consecución de pacientes, análisis de resultados y elaboración del manuscrito.

Silvia Duarte: ejecución experimental de la segunda fase del estudio, análisis de resultados, preparación de gráficas y contribución a la elaboración del manuscrito.

Camila Calderón: diseño del estudio, estandarización de las técnicas y ejecución experimental de la primera fase del estudio. John Mario González: asesoría de la estandarización de las técnicas moleculares, seguimiento de la estandarización, dirección del laboratorio donde se realizaron las pruebas, análisis de resultados, elaboración del manuscrito.

Adriana Cuéllar: análisis de resultados y elaboración del manuscrito.

Alberto Gómez: análisis de resultados y elaboración del manuscrito.

Evelyne Halpert: diseño del estudio, consecución de pacientes y análisis de resultados.

Adriana Rodríguez: diseño del estudio, consecución de recursos financieros de la Universidad Militar Nueva Granada, dirección general del estudio, administración de recursos financieros, asesoría en la ejecución experimental, análisis de resultados y elaboración del manuscrito.
} 
with no history of the disease. All children were under 12 years old. RNA was extracted with trizol and the expression levels of cytokines were analyzed by real time PCR technique.

Results: A wide range in the expression levels of IFN-y and IL-10 was noted as well as constant low values of IL-4. Three distinct profiles were observed, but which did not correspond to a recognizable pattern among the patients. The samples obtained from healthy tissues showed no expression of any of the cytokines.

Conclusions: This is the first characterization of cytokines that mediate the immune response at the site of the skin lesion in children with papular urticaria by flea bite. The data indicated that the local response was mixed and that a single phenotype is not predominant among the patients.

Key words: urticaria, cytokines, interleukin-4, interleukin-10, gamma interferon.

La urticaria papular ha sido definida tradicionalmente como una enfermedad alérgica crónica, causada por la exposición a ectoparásitos. Sin embargo, las investigaciones realizadas a la fecha no muestran una clara relación de esta entidad con las enfermedades alérgicas comunes.

En estudios previos realizados en 45 pacientes por nuestro grupo de investigación en urticaria papular por picadura de pulga, se encontró que la población estudiada no reportaba antecedentes familiares o personales de atopia, las pruebas cutáneas de alergia frente al extracto de pulga eran negativas y había una baja asociación con las enfermedades alérgicas comunes. En las biopsias de piel humana a partir de las diferentes lesiones clínicas, se observó un infiltrado con predominio de células TCD4+ y de eosinófilos, y una respuesta aumentada mediada por lgE sérica frente a las proteínas de extracto de pulga completa, especialmente las de 30 a $35 \mathrm{kDa}$ de peso molecular, con relación al grupo control (1).

Aunque no existe claridad con respecto a la patogenia de la urticaria papular, la respuesta de anticuerpos $\lg E$ e $\lg G$ (1), la dinámica de participación de los isotipos de $\lg G$ de acuerdo con el tiempo de evolución de la enfermedad (2) y el aparente estado atópico de los pacientes de acuerdo con el balance Th1/Th2 (3), indican la participación de la respuesta inmunitaria sistémica en la manifestación de la enfermedad y que la reacción inmunitaria frente a las proteínas de la pulga es compleja e involucra más de un mecanismo.

Sin embargo, teniendo en cuenta la importancia de los diferentes microambientes tisulares en la regulación de la respuesta inmunitaria, nos formulamos la siguiente pregunta: ¿cuáles son los

$\overline{\text { Correspondencia: }}$

Elizabeth García, Fundación Santa Fe de Bogotá, Carrera 9 № 117-20, oficina 205, Bogotá, D.C., Colombia

Teléfono: (571) 2152852

eligarcia.gomez@gmail.com

Recibido: 15/04/11; aceptado:12/07/11 niveles de expresión de las citocinas IFN- $\gamma$, IL-10 e IL-4, como marcadoras de la respuesta efectora de células $T$ en las lesiones activas de piel de niños con urticaria papular por picadura de pulga?

Por lo anterior, el objetivo de este trabajo fue analizar la expresión de las citocinas efectoras de la respuesta inmunitaria en las lesiones de piel de pacientes con urticaria papular por picadura de pulga, para lo cual se utilizó la técnica de reacción en cadena de la polimerasa en tiempo real (RTPCR), con el fin de precisar los niveles de expresión de cada una de ellas. Ésta es la primera descripción de citocinas que median la respuesta inmunitaria a nivel local en lesiones activas de niños con urticaria papular por picadura de pulga.

\section{Materiales y métodos}

\section{Muestras}

Se diseñó un estudio exploratorio en el que se tomaron 14 biopsias de $2 \mathrm{~mm}$ de lesiones de piel de niños de ambos sexos, menores de 12 años, con diagnóstico clínico de urticaria papular por picadura de pulga, que asistieron a los Servicios de Dermatología y Alergia Pediátricas de la Fundación Santa Fe de Bogotá. Además, como control, se obtuvo piel sana de 5 niños sin antecedentes de este tipo de urticaria, menores de 12 años de edad, sometidos a otro tipo de cirugía, como herniorrafia. Todos los pacientes, sanos y enfermos, aceptaron voluntariamente participar en el estudio mediante la firma del consentimiento informado por parte del representante legal. Este proyecto fue avalado por el Comité de Ética de la Fundación Santa Fe de Bogotá.

\section{Extracción de ARN de piel}

El aislamiento del ARN total de las muestras de biopsias de pacientes con urticaria papular por picadura de pulga, se hizo con el método de extracción fenólica con trizol (Invitrogen Life Technologies, Carlsbad, CA, USA), siguiendo el protocolo publicado por Chomczynsky en 1987 (4). Como valor indicativo aproximado de la cantidad 
de ARN por utilizar en la RT-PCR, se calculó la concentración por espectofotometría con el equipo SmartSpec® Plus Spectrophotometer (Bio-Rad Laboratories, Hercules, CA, USA). La integridad del ARN se evidenció mediante un corrido electroforético en gel de agarosa al 1,5\% y coloración con bromuro de etidio.

\section{Reacción en cadena de la polimerasa para síntesis del ADN}

Se sintetizó el ADNc utilizando el estuche de aislamiento de cDNA Superscript First Strand cDNA® (Invitrogen Life Technologies, Carlsbad, CA, USA), siguiendo las indicaciones del fabricante, así: $100 \mathrm{ng}$ de ARN, $1 \mu \mathrm{l}$ de dNTP y $0,5 \mathrm{ng} / \mu \mathrm{l}$ de oligonucleótidos al azar. Inicialmente, se incubó la reacción a $65{ }^{\circ} \mathrm{C}$ por cinco minutos $\mathrm{y}$, posteriormente, se agregaron $4 \mu \mathrm{l}$ de solución tampón 10X RT, $2 \mu \mathrm{l}$ de solución mixta de dNTP $10 \mathrm{Mm}, 1 \mu \mathrm{l}$ de la enzima transcriptasa inversa (RT Invitrogen Life Technologies, Carlsbad, CA, USA) ajustada a $10 \mathrm{U}$. Se incubó una hora a $42^{\circ} \mathrm{C}$, en un termociclador (MJ Mini Personal Thermal Cycler ${ }^{\circledR}$, BioRad Laboratories, Hercules, CA, USA).

\section{Reacción en cadena de la polimerasa en tiempo} real

Como gen constitutivo, se utilizó el gen de la hipoxantina-guanina-fosforribosil-transferasa (HGPRT). Para la RT-PCR se utilizó FastStart® TaqMan qRT-PCR master mix, que contiene todos los reactivos, excepto los oligonucleótidos cebadores y muestra. La reacción se estandarizó con las siguientes condiciones: FastStart TaqMan® Probe Master, Hydrolysis Probe (12,5 $\mu$ l), oligonucleótidos cebadores forward y reverse del gen constitutivo y de cada una de las citocinas $200 \mathrm{mM}(0,3 \mu \mathrm{l})$, sonda del gen constitutivo y de cada una de las citocinas 250 $\mathrm{mM}$ y de ADNc $(7,5 \mathrm{ng} / \mu \mathrm{l})$. El volumen se completó a $45 \mu \mathrm{l}$ con agua ultrapura de grado PCR.

Las secuencias de los oligonucleótidos cebadores y de las sondas conjugadas con diferentes fluorocromos, utilizadas para la detección de cada una de las citocinas (5) y el gen constitutivo (6), fueron las siguientes:

IL-4(149 pb)

FW:5'CCACGGACACAAGTGCGATA-3'

RV:5'CCCTGCAGAAGGTTTCCTTCT-3'

Sonda:5'TCTGTGCACCGAGTTGACCG TAACAGAC-3. Cal Flúor red 610

IL-10 (138 pb)

FW: 5'-GTGATGCCCCAAGCTGAGA-3'
RV: 5'-CACGGCCTTGCTCTCTGTTTTT-3

Sonda:5'-CCAAGACCCAGACATCAAGGCGCA-3. Cal flúor orange 560

IFN-y (120 pb)

FW: 5'-TCAGCTCTGCATCGTTTTGG -3

RV: 5'-GTTCCATTATCCGCTACATCTGAA -3'

Sonda: 5'TTGGCTGTTACTGCCAGGACCCATAT GT-3. Quasar 707

HGPRThipoxantina-guanina-fosforribosil-transferasa (101 pb)

FW: 5'-GACTTTGCTTTCCTTGGTCAGG-3'

RV: 5'-AGTCTGGCTTATATCCAACACTTCG-3

Sonda: 5'TTTCACCAGCAAGCTTGCGACCTTGA $-3^{\prime}$. FAM

Las tres citocinas se corrieron bajo el mismo protocolo, excepto por las temperaturas de alineamiento, así: programa de desnaturalización, $95{ }^{\circ} \mathrm{C}$ cinco minutos, programa de amplificación y cuantificación, $94{ }^{\circ} \mathrm{C}$ por 20 segundos, $68^{\circ} \mathrm{C}$ por 45 segundos y $72{ }^{\circ} \mathrm{C}$ por 50 segundos con 35 ciclos. Las temperaturas de alineamiento fueron de $55^{\circ} \mathrm{C}$ para IL-10 y de $60^{\circ} \mathrm{C}$ para IFN-y e IL-4. Para la cuantificación, se utilizaron los valores correspondientes al primer ciclo de amplificación del templado, relativos al gen normalizador de referencia HPRT, utilizando la siguiente fórmula: $2^{-\Delta C T}(\triangle C T=C T$ del gene de interés - CT normalizador) (7).

\section{Análisis de la información}

Los resultados se presentan de manera descriptiva, como medianas y percentiles 25 y 75 . Para la comparación de los niveles de expresión de las citocinas estudiadas, se usó la prueba $U$ de MannWhitney, con un nivel de significancia menor de 0,05 .

\section{Resultados}

Se hizo un análisis de cuantificación relativa de la expresión de los genes de IL-10, IL-4 e IFN-y. Se encontraron niveles de las tres citocinas estudiadas: IFN-y (mediana=163,5; percentil 25: 67,75 y percentil 75 : 38320 ), IL-10 (mediana $=166$; percentil 25: 97,75 y percentil 75: 21510) e IL-4 (mediana=49; percentil 25: 27 y percentil 75: 64). Los niveles de expresión de ADNc de las citocinas IFN-y e IL-10, mostraron una gran variabilidad en el grupo, mientras que, para IL-4 se encontró que los niveles de expresión se mantuvieron constantes en todos los pacientes (figura 1). La comparación entre las tres citocinas estudiadas mostró una expresión significativamente más baja de IL-4 con respecto a IL-10 ( $p=0,0003)$ e IFN- $y(p=0,0008)$ (figura 1). 
Las 14 muestras se pudieron agrupar según la producción de las citocinas, en tres perfiles diferentes. El primer grupo correspondió a $36 \%$ $(n=5)$ de las biopsias que presentaron niveles aumentados de IL-10 y niveles bajos de IL-4 e IFN-y (figura 2A). El segundo grupo correspondió a $36 \%(n=5)$, y mostró niveles altos de IFN-y y bajos de IL-4 e IL-10 (figura 2B). En el tercer grupo de cuatro biopsias (28\%), los niveles fueron bajos para las tres citocinas (figura 2C). Estos perfiles no presentaron una relación con edad, tiempo de evolución o antecedentes personales y familiares de alergia de los pacientes.

En cuanto a las biopsias de individuos sanos, en ninguna de las muestras procesadas se encontró expresión de las citocinas estudiadas.

\section{Discusión}

Existe abundante información que indica que las citocinas presentes en la piel influyen en los fenómenos inmunológicos, como activación, patrones de migración, quimiotaxis, diferenciación y proliferación (8). En la piel sana se encuentra, aproximadamente, el doble de células $\mathrm{T}$ que en la sangre periférica (9). Esto, sumado al hecho de que los individuos receptores de trasplantes que reciben medicamentos inmunosupresores tienen 65 a 250 veces mayor riesgo de desarrollar carcinomas de células escamosas (10), sugiere que existe un mecanismo eficiente de inmunovigilancia en la piel, aun en ausencia de estímulos inflamatorios.

El paradigma descrito en 1986 por Coffman y Mosmann, propone que las reacciones de hipersensibilidad retardada y que la actividad ayudadora para la inmunidad humoral dependen de dos tipos diferentes de células T CD4+, en virtud

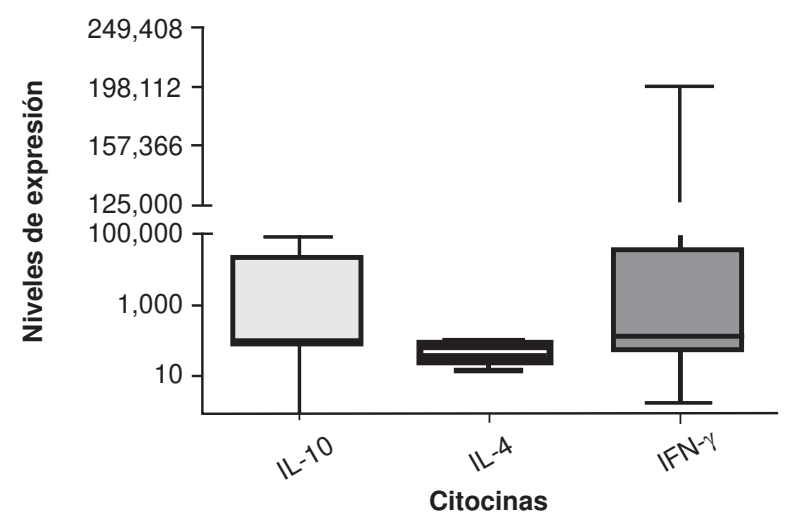

Figura 1. Niveles de expresión de IL-4, IL-10 e IFN- $\gamma$, con respecto al gen constitutivo hipoxantina guanina fosforribosil transferasa (HGPRT) en las muestras de 14 pacientes con urticaria papular por picadura de pulga. Los resultados se presentan en medianas y percentiles 25 y 75 . del tipo de citocinas secretadas por estas células $(11,12)$. Asimismo, se describió que la activación adecuada de las células $\mathrm{T}$ induce un proceso de diferenciación que resulta en la polarización de la respuesta linfoide a Th1 o Th2, de acuerdo con el tipo de citocinas secretadas, como IFN-y o IL-4, respectivamente.

Aunque este patrón de respuesta se ha estudiado en diferentes enfermedades, se han demostrado claras diferencias en la respuesta inmunitaria local y sistémica estudiada en lasangre periférica. En la sangre, las células T CD4+ Th1 y Th2 se encuentran normalmente en una proporción 7:1 (13), mientras que en la piel sana, tomada de pacientes adultos sometidos a lipectomía, se encontró que $95 \%$ de las células T CD4+ se encuentran polarizadas hacia el fenotipo Th1 (9). A diferencia de los adultos, los estudios previos realizados en piel sana de niños sin antecedentes de urticaria papular por picadura de pulga, no encontraron un infiltrado inflamatorio (1), lo cual sustenta el hallazgo de la ausencia de las citocinas analizadas en este estudio en la piel sana de los niños del grupo control.

En cuanto al papel de las citocinas descrito en enfermedades inflamatorias de la piel, se ha propuesto que el proceso inflamatorio depende no sólo del balance Th1/Th2, sino, además, y de forma importante, de la participación de las células Th17 en enfermedades como la dermatitis atópica (14) y la dermatitis de contacto (15). El papel de las células Th17 en la respuesta local de la urticaria papular por picadura de pulga, no se ha determinado hasta el momento.

Además, se han descrito otros fenotipos efectores de linfocitos T CD4+ con función reguladora y que tienen un papel importante en la regulación de la respuesta inflamatoria alérgica (16). En estas poblaciones celulares se encuentran células $T$ reguladoras naturales, en las cuales se ha descrito actividad en enfermedades alérgicas como la dermatitis atópica (17). Otras poblaciones de células $T$ reguladoras inducidas por el estímulo antigénico, se caracterizan por la secreción de citocinas reguladoras de la respuesta inmunitaria, como $\mathrm{IL}-10$ o factor beta de crecimiento transformador (TGF- $\beta$ ) (18).

Algunos estudios sobre la evaluación de las citocinas de las células $T$ obtenidas de lesiones de pacientes con dermatitis de contacto a níquel, informan la producción de altos niveles de IL-5 y niveles variables de IFN-y e IL-4, con predominio de la respuesta Th2 (19). Sin embargo, en otros estudios se sugiere que estos individuos tienen un 
A)

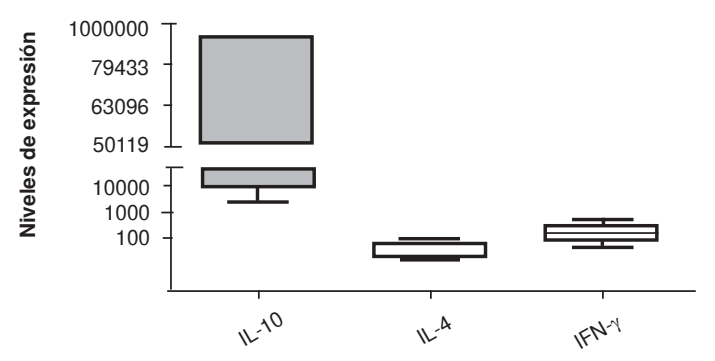

C)
B)

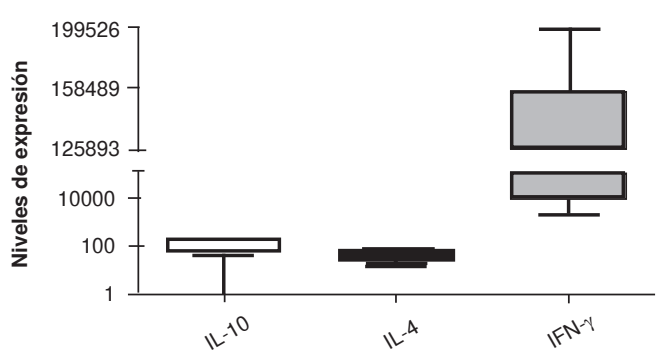

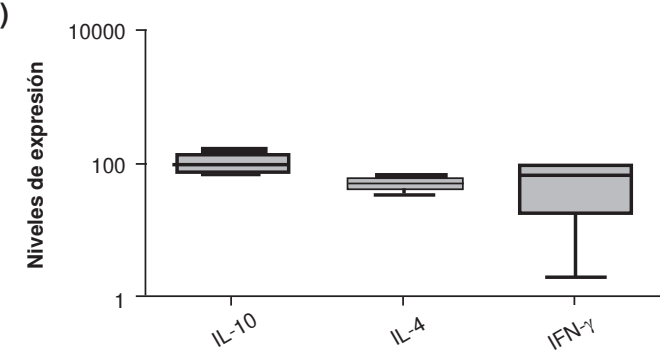

Figura 2. Perfiles de niveles de expresión de citocinas en lesiones activas de pacientes con urticaria papular por picadura de pulga. A. Cinco muestras presentaron alta expresión de IL-10 y baja expresión de IFN-y B. Cinco muestras presentaron niveles altos de IFN-y y baja expresión de IL-10. C. Cuatro muestras presentaron niveles similares de expresión de las tres citocinas analizadas. Los resultados se presentan en medianas y percentiles 25 y 75.

patrón de respuesta con predominio de Th1 con mayor producción de IFN-y (20), lo cual indica que puede haber una respuesta variable en cuanto al balance Th1/Th2 en la patogenia de la dermatitis de contacto.

El estudio de los mecanismos involucrados en la iniciación y mantenimiento de la inflamación en la piel de pacientes con dermatitis atópica, muestra que a nivel local se expresan abundantemente IL-4 e IL-5, citocinas asociadas al fenotipo Th2, aunque en las lesiones agudas se encuentra predominantemente IL-4, mientras en las lesiones crónicas predomina la IL-5 (21).

Aunque en estudios previos sobre la respuesta inmunitaria en pacientes con urticaria papular por picadura de pulga, se sugiere una respuesta con predominio de Th2 a nivel local, en virtud de un infiltrado en el que predominan las células TCD4+ y los eosinófilos (1).

Los resultados obtenidos en este trabajo muestran que no se encuentra un predominio claro de citocinas a nivel local en todos los pacientes que pueda sugerir un patrón Th1 (IFN- $\mathrm{\gamma}$ ), Th2 (IL-4) 0 de citocinas reguladoras (IL-10). El predominio de eosinófilos en los infiltrados inflamatorios, es una demostración indirecta del predominio de la respuesta Th2; sin embargo, desde el punto de vista biológico y dada la heterogeneidad de la respuesta linfoide inducida por un estímulo antigénico, es posible que el mecanismo involucrado en la reacción inflamatoria esté definido por la actividad que ejercen las citocinas presentes, más probablemente relacionado con la calidad de un tipo de respuesta que por la cantidad de la misma.

Actualmente, el tratamiento para la enfermedad alérgica incluye esquemas de corta duración con antihistamínicos o corticoesteroides, que inducen una supresión temporal de la alergia, y el único tratamiento que induce tolerancia por largos periodos es la inmunoterapia, la cual se ha relacionado con el aumento de citocinas reguladoras (22). Después de la inmunoterapia se ha mostrado una desviación de la respuesta inmunitaria con supresión de la proliferación de células $T$, acompañada por aumento en la secreción de IL-10 y TGF- $\beta$ por células T específicas para el alérgeno, así como aumento en el número de células reguladoras (23). Según Jutel, después de la inmunoterapia, la producción de IL-10 y TGF- $\beta$ aumenta a niveles similares a los encontrados en individuos sanos frente al mismo alérgeno, como un mecanismo que restaura la tolerancia (24).

Los hallazgos obtenidos en este trabajo demuestran que, en los pacientes con mayor expresión de IL-10, los niveles de IFN- $\gamma$ fueron menores, mientras que los pacientes con gran expresión de IFN- $\gamma$, tenían menores niveles de expresión 
de IL-10. Previamente, nuestro grupo demostró que la estimulación de linfocitos T CD4+ de sangre periférica refleja un predominio de células secretoras de IL-4, al comparar con células de controles sanos en las que se observó predominio de linfocitos secretores de IFN-Y (3).

Estos hallazgos, sumados a la variabilidad encontrada a nivel local en las lesiones de los pacientes, nos permiten sugerir que en la urticaria papular por picadura de pulga, los pacientes tienen un estado atópico basal con predominio de la respuesta de tipo Th2 a nivel sistémico, que no se ve reflejado a nivel local, dado que las muestras obtenidas para las dos mediciones se tomaron en el mismo momento en el mismo individuo.

Debido a que la frecuencia de picaduras por pulgas es un parámetro de difícil control en la población pediátrica, es posible que aquellos individuos que hayan estado expuestos a una mayor cantidad de antígenos de pulga, similar a lo que podría ocurrir en una inmunoterapia, tengan la capacidad de producir más IL-10 a nivel local con un efecto regulador sobre las células Th1 y, probablemente, sean estos los pacientes más cercanos a la tolerancia a los antígenos de pulga, fenómeno observado en la mayoría de los pacientes con urticaria papular por picadura de pulga. Y, aunque la manifestación clínica de la enfermedad sea similar, los mecanismos de regulación de la respuesta inmunitaria en los diferentes individuos varían de acuerdo con el fondo genético, la frecuencia del contacto con el antígeno inductor y la cantidad de antígeno presente en cada contacto.

Los datos aquí reportados corresponden a un estudio exploratorio en el que se describen las citocinas que median la respuesta inmunitaria en el sitio de la lesión cutánea en niños con urticaria papular por picadura de pulga,lo que indica que en la respuesta local en piel hay acumulación de, al menos, tres citocinas (IL-10, IL-4 e IFN- $\mathrm{Y}$ ), aunque no se encuentra predominio de un perfil común de la respuesta efectora de las células $T$.

Teniendo en cuenta la dificultad para la toma de las muestras de piel de niños enfermos y sanos, es necesario diseñar futuros estudios que analicen la expresión de marcadores celulares en piel, como el antígeno CLA en células de sangre periférica.

\section{Agradecimientos}

A Armando Rojas, por su contribución para la consecución de las biopsias de piel sana de los niños del grupo control.

\section{Conflicto de interés}

Los autores declaramos que no existen conflictos de interés en la ejecución de esta investigación ni en la elaboración del artículo.

\section{Financiación}

Esta investigación fue financiada por la Universidad Militar Nueva Granada, bajo el código MED 051, y por la Fundación Santa Fe de Bogotá. Fue cofinanciada por la Pontificia Universidad Javeriana y la Universidad de los Andes.

\section{Referencias}

1. García E, Halpert E, Rodríguez A, Andrade R, Fiorentino S, García C. Immune and histopathologic examination of flea bite-induced papular urticaria. Ann Allergy Asthma Immunol. 2004;92:446-52.

2. Cuéllar A, Rodríguez A, Halpert E, Rojas F, Gómez A, Rojas A, et al. Specific pattern of flea antigen recognition by $\lg G$ subclass and $\lg E$ during the progression of papular urticaria caused by flea bite. Allergol Immunopathol. 2010;38:197-202.

3. Cuéllar A, Rodríguez A, Rojas F, Halpert E, Gómez A, García E. Differential Th1/Th2 balance in peripheral blood lymphocytes from patients suffering from flea biteinduced papular urticaria. Allergol Immunopathol (Madrid). 2009;37:7-10.

4. Chomczynski P, Sacchi N. Single-step method of RNA isolation by acid guanidinium thiocyanate-phenol-chloroform extraction. Anal Biochem.1987;162:156-9.

5. Giulietti A, Overbergh L, Valckx D, Decallonne B, Bouillon R, Mathieu C. An overview of real-time quantitative PCR: Applications to quantify cytokine gene expression. Methods. 2001;25:386-401.

6. Bilsborough $\mathbf{J}$, Leung $\mathbf{D}$, Maurer $\mathbf{M}$, Howell $\mathbf{M}$, Boguniewcz M, Yao L, et al. IL-31 is associated with cutaneous lymphocyte antigen-positive skin homing $T$ cells in patients with atopic dermatitis. J Alllergy Clin Immunol. 2006;117:418-25.

7. Thomas D. Analyzing real-time PCR data by the comparative CT method. Nat Protoc. 2008;3:1101-8.

8. Roggen EL, Lindstedt M, Borrebaeck C, Verheyen GR. Interactions between dendritic cells and epithelial cells in allergic disease. Toxicol Lett. 2006;162:71-82.

9. Clark RA, Chong B, Mirchandani N, Brinster NK, Yamanaka K, Dowgiert RK, et al. The vast majority of CLA+ $T$ cells are resident in normal skin. J. Immunol. 2006;176:4431-9.

10. Berg D, Otley CC. Skin cancer in organ transplant recipients: Epidemiology, pathogenesis, and management. J Am Acad Dermatol. 2002;47:1-17.

11. Coffman RL, Carty J. A T cell activity that enhances polyclonal $\lg \mathrm{E}$ production and its inhibition by interferongamma. J Immunol. 1986;136:949-54.

12. Mosmann TR, Cherwinski H, Bond MW, Giedlin MA, Coffman RL. Two types of murine helper T cell clone. I. 
Definition according to profiles of lymphokine activities and secreted proteins. J Immunol. 1986;136:2348-57.

13. Teraki Y, Picker LJ. Independent regulation of cutaneous lymphocyte-associated antigen expression and cytokine synthesis phenotype during human CD4+ memory $\mathrm{T}$ cell differentiation. J Immunol. 1997; 159:6018-29.

14. Matsushita S, Takagi R, Hashimoto K, Higashi T. Qualitative evaluation of adjuvant activities and its application to Th2/17 diseases. Int Arch Allergy Immunol. 2011;155(Suppl.1):2-5.

15. Larsen JM, Bonefeld CM, Poulsen SS, Geisler C, Skov L. IL-23 and $\mathrm{T}(\mathrm{H}) 17$-mediated inflammation in human allergic contact dermatitis. J Allergy Clin Immunol. 2009;123:486 92.

16. Braga M, Quecchia C, Cavallucci E, Di Giampaolo L, Schiavone C, Petrarca $\mathbf{C}$, et al. T regulatory cells in allergy. Int J Immunopathol Pharmacol. 2011;24(Suppl.):55S-64S.

17. Reefer AJ, Satinover SM, Solga MD, Lannigan JA, Nguyen JT, Wilson BB, et al. Analysis of CD25hiCD4+ "regulatory" T-cell subtypes in atopic dermatitis reveals a novel $\mathrm{T}(\mathrm{H}) 2$-like population. J Allergy Clin Immunol. 2008;121:415-22.

18. Bluestone J, Mackay C, O'Shea J, Stockinger B. The functional plasticity of $\mathrm{T}$ cell subsets. Nat Rev Immunol. 2009;9:811-6.
19. Probst $\mathbf{P}$, Küntzlin $\mathbf{D}$, Fleischer $\mathbf{B}$. TH2-type infiltrating T cells in nickel-induced contact dermatitis. Cell Immunol. 1995;165:134-40.

20. Kapsenberg ML, Wierenga EA, Stiekema FE, Tiggelman AM, Bos JD. Th1 lymphokine production profiles of nickelspecific CD4+T-lymphocyte clones from nickel contact allergic and non-allergic individuals. J Invest Dermatol. 1992;98:59-63.

21. Hamid Q, Boguniewicz M, Leung DY. Differential in situ cytokine gene expression in acute versus chronic atopic dermatitis. J Clin Invest. 1994;94:870-6.

22. Akdis M, Verhagen J, Taylor A, Karamloo F, Karagiannidis C, Crameri R, et al. Immune responses in healthy and allergic individuals are characterized by a fine balance between allergen-specific $T$ regulatory 1 and $T$ helper 2 cells. J Exp Med. 2004;199:1567-75.

23. Francis J, Hill S, Durham S. Induction of IL-10+CD4+CD25+ $\mathrm{T}$ cells by grass polen immunotherapy. J Allergy Clin Immunol. 2003;111:1255-81.

24. Jutel M, Akdis M, Budak F, Aebischer-Casaulta C, Wrzyszcz M, Blazer K, et al. IL-10 and TGF $\beta$ cooperate in the regulatory $T$ cell response to mucosal allergens in normal immunity and specific immunotherapy. Eur $\mathrm{J}$ Immunol. 2003;33:1205-14. 